\title{
THE LANCZOS POTENTIAL FOR THE WEYL CURVATURE TENSOR: EXISTENCE, WAVE EQUATION AND ALGORITHMS.
}

\author{
S. Brian Edgar and A. Höglund \\ Department of Mathematics, Linköping University, \\ Linköping, \\ S58183 Sweden.
}

\begin{abstract}
In the last few years renewed interest in the 3 -tensor potential $L_{a b c}$ proposed by Lanczos for the Weyl curvature tensor has not only clarified and corrected Lanczos's original work, but generalised the concept in a number of ways. In this paper we carefully summarise and extend some aspects of these results, and clarify some misunderstandings in the literature. We also clarify some comments in a recent paper by Dolan and Kim; in addition, we correct some internal inconsistencies in their paper and extend their results.

The following new results are also presented. The (computer checked) complicated second order partial differential equation for the 3-potential, in arbitrary gauge, for Weyl candidates satisfying Bianchi-type equations is given - in those $n$-dimensional spaces (with arbitrary signature) for which the potential exists; this is easily specialised to Lanczos potentials for the Weyl curvature tensor. It is found that it is only in 4-dimensional spaces (with arbitrary signature and gauge), that the non-linear terms disappear and that the awkward second order derivative terms cancel; for 4-dimensional spacetimes (with Lorentz signature), this remarkably simple form was originally found by Illge, using spinor methods. It is also shown that, for most 4-dimensional vacuum spacetimes, any 3-potential in the Lanczos gauges which satisfies a simple homogeneous wave equation must be a Lanczos potential for the Weyl curvature tensor of the background vacuum spacetime. This result is used to prove that the form of a possible Lanczos potential proposed by Dolan and Kim for a class of vacuum spacetimes is in fact a genuine Lanczos potential for these spacetimes.
\end{abstract}




\section{Introduction}

Although the existence of a 3-tensor $L_{a b c}$ as a potential for the Weyl conformal tensor $C_{a b c d}{ }^{\dagger}$ in 4-dimensional spacetimes was first suggested by Lanczos (1962), for some time there was little significant development probably due to some mistakes and misunderstandings, both in the original paper, and in some subsequent papers. However, more recently a number of interesting results have been obtained. There are a number of subtleties in these results — involving dimension, signature, gauge, and indeed the class of tensors for which potentials can be found - and again a number of misunderstandings and mistakes have crept into the literature; so we shall first present a careful summary of these results.

The topic was placed on a firm foundation and in a wider context by Bampi and Caviglia (1983); they detected a logical flaw in Lanczos's original argument, and not only gave a valid and rigorous proof of existence, but extended Lanczos's original proposal to a larger class of 4-tensors, to a larger class of 3-tensors, and to a larger class of spaces. Bampi and Caviglia have shown - in any 4-dimensional analytic manifold with metric $g_{a b}$ (irrespective of signature) — that any analytic 4-tensor $W_{a b c d}$ with the properties

$$
\begin{array}{r}
W_{a b c d}=W_{[a b] c d}=W_{a b[c d]} \\
W_{b a d}^{a}=0=W_{a[b c d]}
\end{array}
$$

always admits locally a regular analytic 3 -tensor potential $L_{a b c}$ with the properties,

$$
\begin{gathered}
L_{a b c}=L_{[a b] c} \\
L_{[a b c]}=0
\end{gathered}
$$

according to

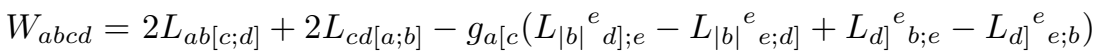

$$
\begin{aligned}
& \left.+g_{b[c}\left(L_{|a|} e^{e} d\right] ; e-L_{|a|} e_{e ; d]}^{e}+L_{d]} e_{a ; e}-L_{d]} e_{e ; a}\right)+\frac{4}{3} g_{a[c} g_{d] b} L^{e f}{ }_{e ; f}
\end{aligned}
$$

(The original presentation by Lanczos (1962) was not in the form (1.3) but rather in an equivalent form using the Hodge dual operator.)

We shall refer to a tensor $W_{a b c d}$ with the properties (1.1) as a Weyl conformal curvature tensor candidate (or Weyl tensor candidate); and obviously any 3-tensor $L_{a b c}$, satisfying (1.2), acts as a potential for some Weyl tensor candidate.

Lanczos had imposed the additional conditions,

$$
L_{a b}^{b}=0 \quad \text { (a) } \quad L_{a b ; c}^{c}=0
$$

$\dagger$ In general, for tensors and spinors, we follow the notation and conventions of Penrose and Rindler (1984); for the Lanczos tensor/spinor we follow the notation of Dolan and Kim (1994a,b) (except for the definitions of the tetrad/dyad components of the Lanczos tensor, as we shall explain in Section 3). We emphasise the importance of care with notations and conventions; this is the cause of some of the previous and present misunderstandings. In particular we point out that in each one of the papers to which we refer in some detail - Lanczos (1962), Bampi and Caviglia (1983), Illge (1988), Novello and Velloso (1987), Zund (1975), Ares de Parga G., et al (1989) — at least one different convention is used compared to those used in this paper. 
and although he had shown directly the admissibility of the first, he only provided a heuristic argument for the admissibility of the second. On the other hand, Bampi and Caviglia showed that these restrictions are not essential, and gave a rigorous proof for the admissibility of any values on the right hand sides of the two equations in (1.4). Since these choices have no effect on the Weyl candidate defined by (1.3) they are gauge choices, and $(1.4 \mathrm{a}, \mathrm{b})$ are referred to respectively as the Lanczos algebraic gauge and the Lanczos differential gauge. ${ }^{\dagger}$.

Illge (1988) has supplied a much simpler existence proof than that in Bampi and Caviglia (1983); however, since this proof is obtained using the spinor formalism of Penrose and Rindler (1984), it is valid only in spacetimes (i.e. four dimensional spaces with Lorentz signature). He has shown that any symmetric 4-spinor $W_{A B C D}$ (Weyl spinor candidate) admits locally a solution to

$$
W_{A B C D}=2 \nabla_{(A} \dot{A} L_{B C D) \dot{A}}
$$

where $L_{B C D \dot{A}}$ is symmetric in all undotted indices (which means that the Lanczos algebraic gauge is built in). If in addition $L_{B C D \dot{A}}$ satisfies the Lanczos differential gauge

$$
\nabla^{A \dot{A}} L_{A C D \dot{A}}=0
$$

then the symmetry brackets in (1.5) may be omitted.

It is also shown in Illge $(1988)^{\dagger}$ that if $W_{A B C D}$ satisfies a homogeneous Bianchi-type equation of the form

$$
\nabla^{A \dot{A}} W_{A B C D}=0
$$

$\dagger$ The status of these conditions is not always made explicit. In Ares de Parga et al. (1989) and López-Bonilla et al. (1993) the Lanczos algebraic gauge (1.4a) is used as part of the definition of the potential with no mention that it is a gauge condition, but the differential gauge condition is not mentioned; while the set of equations (1.3) is given in an abreviated form, with the Lanczos algebraic gauge condition (1.4a) substituted. In Dolan and Kim (1994a,b) the condition (1.4a) is also used as part of the definition of the potential with no mention that it is a gauge condition, but the Lanczos differential gauge condition (1.4b) is used, and explicitly described as such; while the set of equations (1.3) is quoted in the most general form without any gauge simplifications. In spinor presentations, the Lanczos algebraic gauge condition is built into the symmetry of the usual Lanczos spinor $L_{A B C \dot{A}}=L_{(A B C) \dot{A}}$, but it would be easy to define a different spinor with an arbitrary algebraic gauge; the differential gauge is not built in, being given explicitly by (1.6), but its imposition means that (1.5) can be written as $W_{A B C D}=2 \nabla_{A} \dot{A} L_{B C D \dot{A}}$

$\dagger$ We emphasise that the results in Illge (1988) are much more general than the extract summarised here: the main theorem is actually given in terms of a Cauchy problem, and both the theorem and the wave equation obtained there are for the case of a symmetric $n$-spinor $W_{A B C D \ldots N}$ in general non-vacuum 4-dimensional spacetimes, with spinor potential in arbitrary differential gauge. 
then $L_{B C D \dot{A}}$ satisfies a homogeneous wave equation, which in vacuum (i.e. Einstein tensor $G_{a b}=0$ ) and in both Lanczos gauges has the remarkably simple form

$$
\square L_{A C D \dot{A}}=0
$$

or equivalently in tensor notation in both Lanczos gauges,

$$
\square L_{a b c}=0
$$

where $\square\left(=\nabla_{a} \nabla^{a}\right)$ is the differential wave operator in four dimensional spacetime.

However, when the analogous analysis is carried through in tensor notation, i.e. for a potential tensor $L_{a b c}$ satisfying Lanczos gauges (1.4), and for a Weyl candidate $W_{a b c d}$ satisfying a homogeneous Bianchi-type equation $W_{a b c}{ }^{d} ; d=0$ in vacuum (i.e. Einstein tensor $G_{a b}=0$ ), we obtain

$$
\nabla^{2} L_{a b c}=2 L^{\text {def }} g_{[a|c|} C_{b] d e f}-2 L_{[a}{ }^{d e} C_{b] e d c}-\frac{1}{2} L^{d e}{ }_{c} C_{d e a b}
$$

where $\nabla^{2}\left(=\nabla_{a} \nabla^{a}\right)$ and $C_{a b c d}$ is the Weyl tensor of the background four dimensional space of arbitrary signature. This appears, at first sight, to be much more complicated than the homogeneous wave equation (1.8b), for the special case of Lorentz signature. However, this apparent disagreement has been resolved in Edgar (1994a) where it is shown that the expression on the right hand side of (1.9), is identically zero in four (and only four) dimensions i.e.

$$
2 L^{\text {def }} g_{[a|c|} C_{b] d e f}-2 L_{[a}{ }^{d e} C_{b] e d c}-\frac{1}{2} L^{d e}{ }_{c} C_{d e a b} \equiv 0
$$

where $L_{a b c}$ is in the algebraic Lanczos gauge. It is emphasised that this identity is a consequence of purely algebraic properties; no differential properties are assumed between $L_{a b c}$ and $C_{a b c d}{ }^{\dagger}$ So therefore, in four dimensional spaces of any signature, $(1.8 \mathrm{~b})$ generalises to

$$
\nabla^{2} L_{a b c}=0
$$

Clearly, since the Weyl conformal tensor $C_{a b c d}$ and its spinor counterpart $\Psi_{A B C D}$, are special cases of the Weyl candidates discussed above, the above results for existence of a potential apply directly to them. We shall reserve the term Lanczos potential for the potential of a Weyl conformal curvature tensor $C_{a b c d}$ (or spinor $\left.\Psi_{A B C D}\right)$ which can therefore also be written, in arbitrary gauges, in four dimensions with arbitrary signature as

$$
\begin{aligned}
& \left.\left.C_{a b c d}=2 L_{a b[c ; d]}+2 L_{c d[a ; b]}-g_{a[c}\left(L_{|b|}{ }^{e} d\right] ; e-L_{|b|}{ }^{e} e ; d\right]+L_{d]}{ }^{e} b ; e-L_{d]}{ }^{e} e ; b\right) \\
& \left.\left.+g_{b[c}\left(L_{|a|}{ }^{e} d\right] ; e-L_{|a|}{ }^{e} e ; d\right]+L_{d]}{ }_{a ; e}-L_{d]}{ }_{e ; a}\right)+\frac{4}{3} g_{a[c} g_{d] b} L^{e f}{ }_{e ; f}
\end{aligned}
$$

$\dagger$ In fact this is a special case of a more general 4-dimensional identity,

$$
2 L^{\text {def }} g_{[a|c|} W_{b] d e f}-2 L_{[a}{ }^{d e} W_{b] e d c}-\frac{1}{2} L^{d e}{ }_{c} W_{d e a b}-\frac{1}{2} W_{a b c d} L^{d e}{ }_{e} \equiv 0
$$

where $L_{a b c}$ is in arbitrary algebraic gauge, and $W_{a b c d}$ is any Weyl candidate, (Edgar and Höglund (1995)). 
Since in vacuum the Weyl tensor satisfies the Bianchi identity in the form,

$$
\nabla^{d} C_{a b c d}=0
$$

the second order equation (1.8c) is also valid, in vacuum, for the Lanczos potential of the Weyl tensor $C_{a b c d}$. The Weyl spinor is given by

$$
\Psi_{A B C D}=2 \nabla_{(A} \dot{A}_{B C D) \dot{A}}
$$

in Lanczos algebraic gauge, and since in vacuum the Weyl spinor satisfies the Bianchi identity in the form,

$$
\nabla^{A \dot{A}} \Psi_{A B C D}=0
$$

the wave equation $(1.8 \mathrm{a})^{\dagger}$ is also valid, in vacuum, for the Lanczos potential of the Weyl spinor $\Psi_{A B C D}$.

In a recent paper Dolan and Kim (1994a) also derive the tensor identity (1.10) in four dimensional spacetimes by spinor methods, and this leads them to make the claims that this identity 'is restricted to Lorentz signature' and also 'that spinor methods seem to be essential to prove all the 4-dimensional identities'. Although Dolan and Kim do refer to the proof for this identity given in Edgar (1994a), they seem to have overlooked the fact that, in that paper, a tensor proof is given, which is explicitly stated and shown to be independent of metric signature; in fact the identity was not verified by the computer algebra system STENSOR as Dolan and Kim state, but rather by a simple tensor manipulation with Hodge duals for four dimensional spaces. In addition, Dolan and Kim have given another identity - (D1) in Dolan and Kim (1994a) — which they also claim to be 'strictly four-dimensional and restricted to Lorentz signature'; but this identity is easily seen to be a direct consequence of the identity (1.10), and so once again signature is irrelevant. $\ddagger$

Dolan and Kim (1994a) also check the accuracy of the non-vacuum wave equation for the Lanczos potential as given by Illge (1988) in both spinor and tensor form. (But it should be noted that, whereas Illge considered

$\dagger$ Although Lanczos (1962) had derived a wave equation for his potential of the Weyl tensor, in four dimensional spacetimes, he had misplaced some of his indices and so his version differs from (1.9); this version has been repeated uncorrected by Atkins and Davis (1980), and has been only partly corrected by Roberts (1989). But it was the work of Illge (1988) in spinors that revealed the very simple form (1.8) of this wave equation, and gave the hint to the existence of the 4-dimensional identity (1.10).

$\ddagger$ These are not the only identities with dimensionally dependent properties which arise in this manner (see Andersson and Edgar (1995)) nor the only time that spinor methods have led to the mistaken conclusion that the signature has some relevance, (see Dianyan (1986)); there has also recently been a related discussion in the context of algebraic invariants of the Riemann tensor, by Jack and Parker (1987), Fulling et al. (1992) and Harvey (1995). Colleagues have drawn our attention to an earlier paper by Lovelock (1970) who shows explicitly that such types of identities are a trivial, but subtle, consequence of dimension alone. In view of this additional interest in such identities, and since other interesting related identities also arise via Lovelock's identities, we give further details separately in Edgar and Höglund (1995). 
potentials in algebraic Lanczos gauge but arbitrary differential gauge of Weyl candidates, Dolan and Kim (1994a) restrict their considerations to potentials in both Lanczos gauges of Weyl curvature tensors; so their check is only valid for potentials in Lanczos gauges of Weyl tensors.) Dolan and Kim confirm that Illge (1988) has given the correct spinor version of the non-vacuum wave equation for the Lanczos potential in Lanczos gauges. They also point out that Illge has omitted a term containing the Ricci scalar $R$ in his translation to the tensor version.

An interesting more general point is that although Lanczos claimed that a potential for the Weyl tensor could only exist in 4-dimensional spacetimes, he gave no rigorous arguments to support this. (However it should be appreciated that his work was in the notation of Hodge duals and so no generalisation to other dimensions was possible within that formalism.) On the other hand the only existence proofs we have, at present, are for four dimensions, given in Bampi and Caviglia (1983) (irrespective of signature) and in Illge (1988) (restricted to Lorentz signature); and in the technicalities of both proofs dimension does play a significant role. As we note in Section 2, it is straightforward to generalise the form of (1.3) to arbitrary $n$ dimensions, but we have no firm evidence either way as to whether such a potential exists for all Weyl tensors in any dimension $n>4$. However, as we shall show in Section 2, the simple homogeneous wave equation (1.8) exists only in four dimensions. In Bampi and Caviglia (1983) there is some discussion for spaces with dimension $n>4^{\dagger}$; however, this is for a parallel existence problem (which is shown to be equivalent to the existence problem for potentials of Weyl candidates only in four dimensions), and so we believe that it is still an open question whether there exists potentials with the properties (1.2) and (1.4) which satisfy the $n$-dimensional generalisation of (1.3) for Weyl tensors in spaces with dimension $n>4$.

Novello and Velloso (1987) have supplied some algorithms for calculating the Lanczos potential of the Weyl conformal curvature tensor in a number of different classes of perfect fluid spacetimes. Their method of establishing these results was simply to substitute into the right hand side of (1.11a) certain proposed forms for the Lanczos potential, and by direct manipulation - a non trivial calculation — show that the Weyl tensor of the background spacetime is obtained. It is emphasised that the Novello and Velloso method is explicitly for Weyl conformal curvature tensors and that they showed explicitly that their resultant 4-tensor

$\dagger$ Bampi and Caviglia (1983) state, for the parallel problem which disregards the cyclic property of the Weyl tensor, that, under generic conditions, a potential can exist only in spaces with dimension $n \leq 6$; this has lead to the careless and incorrect statement that the Lanczos potential for a Weyl tensor exists only in dimensions $4 \leq n \leq 6$ in Roberts (1989), Edgar (1994a), Edgar (1994b). Firstly, there seems to be a simple computational mistake in the very last step of the argument so that the result of Bampi and Caviglia for the parallel problem should instead be $n \leq 5$; secondly it does not seem obvious that this parallel problem is equivalent to the original existence problem for the potential of a Weyl candidate in 5 dimensions; thirdly a generic result for Weyl candidates cannot be directly applied to all Weyl curvature tensors. 
was such a tensor. The work in Novello and Velloso (1987) was, in general, for an arbitrary gauge, although in some cases the form proposed for $L_{a b c}$ satisfies the Lanczos algebraic gauge; in other cases a simple modification

$$
L_{a b c} \rightarrow L_{a b c}-2 g_{c[a} L_{b] e}^{e} / 3
$$

always yields a Lanczos potential which satisfies the algebraic Lanczos gauge. When their algorithms were applied to particular metrics, it was often found that the Lanczos differential gauge was also satisfied. However, as pointed out in Novello and Velloso (1987), there is no compelling argument, in general, to prefer the Lanczos differential gauge.

In the second of their recent papers, Dolan and Kim (1994b) have used and developed some of the results of Novello and Velloso; we feel that some points in this paper require further comment, and we deal with these points later in this paper.

In the next section we present, for the first time, the most general form of the second order differential equation for the 3-potential of a Weyl candidate which satisfies a Bianchi-type equation - in $n$-dimensional spaces with arbitrary signature, and with potential in arbitrary gauge - for such spaces for which such a potential exists; from this we can deduce, also for the first time, the form in arbitrary four dimensional spaces with arbitrary signature and with potential in arbitrary gauge. (Since these calculations have been carried out by computer we hope it will end the need for further checks.) We show explicitly that for Lanczos potentials of Weyl tensors the non-linear complications disappear and the awkward second order terms cancel only in four dimensional spaces (irrespective of signature); further a remarkably simple form of this four dimensional equation seems to occur only in the Lanczos gauges, and the Lanczos-Illge wave equation (1.8) is the vacuum version of this for Lorentz signature. We also confirm that the non-vacuum tensor form for the wave equation of the Lanczos potential in Lanczos gauges given in Dolan and Kim (1994a) is correct, and agree with them that Illge's version has a missing term involving the Ricci scalar; however, contrary to what is claimed in Dolan and Kim (1994a), we have found that this is the only error in the tensor version in Illge (1988). (We have also checked the more general tensor version, in arbitrary differential gauge, as given by Illge (1988).)

In Section 3 we deal directly with some points from the second of the papers by Dolan and Kim (1994b). In that paper they suggest three possible classes of Lanczos potentials (for Weyl tensors) in Lanczos gauges, and propose that 'when these are verified for each spacetime to which they are applied', then they will be genuine Lanczos potentials in Lanczos gauges. However, one of these classes is simply a subclass of a class already shown in Novello and Velloso (1987) to always give Lanczos potentials in Lanczos gauges; so no further explicit verification is necessary. A second of these classes is precisely the vacuum subclass of a class already shown in Novello and Velloso (1987) to always give Lanczos potentials - although not necessarily in Lanczos gauges; so only the gauge conditions need to be explicitly verified. The remaining class suggested 
in Dolan and Kim (1994a) is a new proposal, and so for this class explicit verification of both the defining equation (1.11a) for Weyl tensors, and for the Lanczos gauges (1.4) is essential - but this is not given in Dolan and Kim (1994b) for the application to the Bondi metric. In Section 5 we explicitly confirm the validity of this potential in general for the class proposed by Dolan and Kim, and in particular for the vacuum Bondi metric.

In Section 3, we also point out an unfortunate structural error in the 'Weyl-Lanczos' relations given by Dolan and Kim (1994b). Their spin coefficient version of (1.11) is inconsistent with their definitions for the tetrad/dyad components of the Lanczos potential components. To maintain internal consistency, as well as agreement with the earlier work in Zund (1975) and in Ares de Parga et al. (1989), we propose that the definitions of the Lanczos potential components (both tetrad and dyad version), in Dolan and Kim (1994b), be adjusted by a factor of $(-1)$. A similar change is also required in the two applications which use these definitions.

In Section 4 we establish the perhaps surprising result that in a background four dimensional vacuum spacetime, the homogeneous wave equation (1.8a) for a 3-tensor $L_{a b c}$ in the Lanczos gauges, is a sufficient condition - in general - for $L_{a b c}$ to be a Lanczos potential (up to a constant factor) of the Weyl curvature tensor of this background spacetime. In Section 5 we use this result to confirm, in a very concise manner, the validity of the new 'possible' Lanczos potential proposed by Dolan and Kim (1994b) - in most vacuum metrics with the appropriate symmetries; this includes their proposed new Lanczos potential for the vacuum Bondi metric. We also investigate whether this potential is valid for any non-vacuum Bondi spaces, but unfortunately find that the only physically interesting generalisation seems to be to vacuum spaces with non-vanishing cosmological term.

Although we shall not deal directly with the results in this paper we draw attention to other recent related work: Hammon and Norris (1993) consider the Lanczos potential, and in particular the question of gauge, in a still more general geometric setting; Torres del Castillo (1995) has obtained Lanczos potentials for a large class of spacetimes, including the Kerr metric, by spinor means; Ares de Parga G., et al (1989) and LópezBonilla, J.L. et al (1993) have obtained expressions for the Lanczos potentials of some special spacetimes, in the spin coefficient formalism of Newman and Penrose (1962). 


\section{The general second order differential equation for the Lanczos potential.}

Although we have no evidence to believe that in $n$ dimensions every Weyl candidate $W_{a b c d}$ satisfying (1.1) can be given locally in terms of a 3-tensor potential $L_{a b c}$ satisfying (1.2), we can of course define a class of Weyl candidates by the $n$-dimensional generalisation of (1.3),

$$
\begin{aligned}
& \left.\left.W_{a b c d}=2 L_{a b[c ; d]}+2 L_{c d[a ; b]}-\frac{2}{(n-2)} g_{a[c}\left(L_{|b|}{ }^{e} d\right] ; e-L_{|b|}{ }^{e} e ; d\right]+L_{d]}{ }^{e} b ; e-L_{d]}{ }^{e} e ; b\right)
\end{aligned}
$$

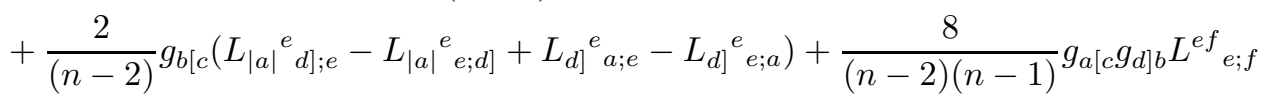

For $n=4$ this expression coincides with (1.3) and is valid for all Weyl candidates; but for $n>4$ we do not know whether the class so defined is the set of all Weyl candidates.

It is assumed that the Weyl candidate satisfies a Bianchi-like equation

$$
W_{a b c d ;}{ }^{d}=J_{a b c}
$$

When we substitute (2.1) in (2.2) we obtain a second order differential equation for the potential $L_{a b c}$ given by

$$
\begin{aligned}
& \nabla^{2} L_{a b c}+\frac{2(n-4)}{n-2} L_{[a}{ }^{d}{ }_{|c ; d| b]}-\frac{3}{(n-2)(n-1)} R L_{a b c} \\
& -2 L_{[b}{ }^{e d} C_{a] d e c}+\frac{1}{2} C_{d e a b} L^{d e}{ }_{c}-\frac{4}{n-2} g_{c[a} C_{b] f e d} L^{f e d} \\
& +\frac{2(n-4)}{n-2} R_{d[a} L_{b]^{d} c}-2 R_{d[a} L_{b] c}{ }^{d}+\frac{n}{n-2} R_{c d} L_{a b}{ }^{d}+\frac{2 n}{(n-2)^{2}} g_{c[a} L_{b] e d} R^{e d} \\
& +G_{a b c} \quad=J_{a b c}
\end{aligned}
$$

where

$$
\begin{aligned}
& G_{a b c}=\frac{1}{n-2}\left(2 g_{c[a} \nabla^{2} L_{b]}^{e} e_{e}+C_{a b c d} L^{d e}{ }_{e}\right. \\
& +\frac{2(n-5)}{n-1} L_{d ; e[b}^{e d} g_{a] c}-2 g_{c[a} L_{b]}^{e d} ; d e
\end{aligned}
$$

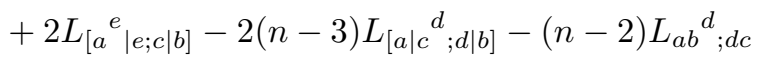

$$
\begin{aligned}
& \left.+\frac{2(n-1)}{n-2} R_{c[a} L_{b] d}{ }^{d}-\frac{2(n-5)}{n-2} g_{c[a} R_{b] e} L_{d}^{e d}-\frac{2(n+1)}{(n-2)(n-1)} g_{c[a} L_{b] d}{ }^{d} R\right)
\end{aligned}
$$

This equation is valid in arbitrary gauge, in arbitrary $n$ dimensions with arbitrary signature; $G_{a b c}$ vanishes when both Lanczos gauges are applied.

We now look at some special cases of this equation, and compare them with existing equations in the literature. We remember that although Illge (1988) gave a tensor version of this equation, this was deduced from his spinor version, and so was strictly only valid in 4-dimensional spacetimes (with Lorentz signature); also the 3-potential of the Weyl candidate was in Lanczos algebraic gauge. Dolan and Kim (1994a), on the otherhand, give both a spinor and an independent 4-dimensional tensor derivation, and so their tensor version is valid for any signature in four dimensions; however, their considerations were explicitly restricted to Lanczos potentials in Lanczos gauges of Weyl curvature tensors. 


\section{(i) Four dimensions.}

In 4-dimensional spaces with arbitrary gauges Edgar and Höglund (1995) show that the identity (1.10) generalises to,

$$
2 L^{\text {def }} g_{[a|c|} C_{b] d e f}-2 L_{[a}{ }^{d e} C_{b] e d c}-\frac{1}{2} L^{d e}{ }_{c} C_{\text {deab }}-\frac{1}{2} C_{a b c d} L^{d e}{ }_{e}=0
$$

Hence in (2.3), in four dimensions, the product terms of $L_{a b c}$ with $C_{a b c d}$ disappear and so do the awkward second derivative terms, because they involve a factor of $(n-4)$, so that $(2.3)$ becomes

$$
\nabla^{2} L_{a b c}-2 R_{d[a} L_{b] c}{ }^{d}+2 R_{c d} L_{a b}^{d}+2 g_{c[a} L_{b] e d} R^{e d}-\frac{1}{2} R L_{a b c}+G_{a b c}=J_{a b c}
$$

where

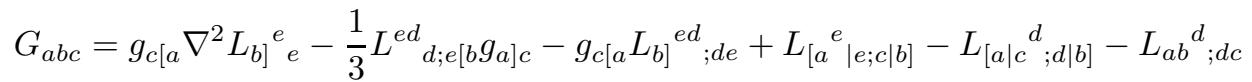

$$
\begin{aligned}
& +\frac{3}{2} R_{c[a} L_{b] d}{ }^{d}+\frac{1}{2} g_{c[a} R_{b] e} L_{d}^{e d}-\frac{5}{6} g_{c[a} L_{b] d}{ }^{d} R
\end{aligned}
$$

So it has now been shown that all product terms of $L_{a b c}$ with $C_{a b c d}$ disappear in any gauge of the Lanczos potential; we have also shown, contrary to what was implied by Dolan and Kim (1994a), that these product terms disappear for all signatures of 4-dimensional spaces.

It may be possible to eliminate $G_{a b c}$ (which contains all the terms arising from arbitrary gauge) by gauge choices other than the two Lanczos gauges, but there are no obvious simple alternatives.

\section{(ii) Four dimensions, Lanczos algebraic gauge.}

Equation (2.6) is unchanged with (2.7) becoming

$$
\left.G_{a b c}=-g_{c[a} L_{b]}^{e d} ; d e-L_{[a \mid c}^{d} ; d \mid b\right]-L_{a b}{ }^{d} ; d c
$$

This agrees with the tensor version (in Lorentz signature) given by Illge (1988) - subject to the addition of the Ricci scalar term, and the different conventions.

\section{(iii) Four dimensions, Lanczos gauges.}

Equation (2.6) becomes

$$
\nabla^{2} L_{a b c}-2 R_{d[a} L_{b] c}{ }^{d}+2 R_{c d} L_{a b}{ }^{d}+2 g_{c[a} L_{b] e d} R^{e d}-\frac{1}{2} R L_{a b c}=J_{a b c}
$$

This of course also agrees with Illge (1988) — subject to the addition of the Ricci scalar term; and when the Weyl candidate $W_{a b c d}$ is specialised to the Weyl curvature tensor $C_{a b c d}$, there is also agreement with the version given by Dolan and Kim (1994a). ${ }^{\dagger}$

$\dagger$ The additional disagreement claimed by Dolan and Kim (1994a), between Illge's tensor equation and their own, is only apparent; it disappears when we take into account the different conventions for the Riemann, Ricci and Lanczos tensors between Dolan and Kim (1994a) and Illge (1988). These are also the conclusions of Illge (1995). 


\section{(iv) Four dimensions, vacuum, Lanczos gauges, Weyl curvature tensor.}

We replace the Weyl candidate $W_{a b c d}$ with the Weyl curvature tensor $C_{a b c d}$, and replace (2.2) with the Bianchi equations so that $J_{a b c}=0$. Equation (2.9) becomes

$$
\nabla^{2} L_{a b c}=0
$$

When we specialise to Lorentz signature we recover the Lanczos-Illge wave equation (1.8b).

(v) $n$-dimensions $(n>4)$.

We have noted in (i) that the expression giving rise to the product terms of $L_{a b c}$ with $C_{a b c d}$

$$
-2 L_{[b}{ }^{e d} C_{a] d e c}+\frac{1}{2} C_{d e a b} L^{d e}{ }_{c}-\frac{4}{n-2} g_{c[a} C_{b] f e d} L^{f e d}+\frac{1}{n-2} C_{a b c d} L^{d e} e_{e}
$$

has been shown to be identically zero in four dimensions. It has also been shown by Höglund (1995) (for Lanczos gauges) and Edgar and Höglund (1995) (for arbitrary gauges) that (2.11) cannot be identically zero for dimensions $n>4 . .^{\dagger}$ We also note that there are terms in (2.3) involving second order derivatives and factors of $(n-4)$ which cannot vanish for dimensions $n>4$. Lanczos gauge choices do not cause any further simplification of the product terms of $L_{a b c}$ with $C_{a b c d}$, nor of the two terms involving second order derivatives, and it is difficult to see any other gauge choices which would be any more successful. It is clear that in dimensions $n>4$ the form of the second order differential equation for the 3-potential of a Weyl candidate has a much more complicated structure than in the 4-dimensional case; and it is remarkable that it is only in four dimensions that such major simplifications occur.

(vi) n-dimensions, vacuum, Lanczos gauges, Weyl curvature tensor.

We replace the Weyl candidate $W_{a b c d}$ with the Weyl curvature tensor $C_{a b c d}$, and replace (2.2) with the Bianchi equations. With the additional vacuum and gauge simplifications, (2.3) simplifies to

$$
\begin{aligned}
& \nabla^{2} L_{a b c}+\frac{2(n-4)}{n-2} L_{[a}{ }^{d}{ }_{|c ; d| b]} \\
& -2 L_{[b}{ }^{e d} C_{a] d e c}+\frac{1}{2} C_{d e a b} L_{c}^{d e}-\frac{4}{n-2} g_{c[a} C_{b] f e d} L^{f e d}=0
\end{aligned}
$$

However, this equation is even more complicated than its appearance suggests; we also have to use (1.11a) to substitute for the Weyl tensor $C_{a b c d}$, and this results in complicated non-linear terms involving products of the Lanczos potential with its first derivatives.

$\dagger$ It is interesting to note that a similar situation arises in the case of the Penrose wave equation for the Weyl tensor; the product terms in this case - involving the Weyl and Ricci tensors - also only disappear in $n=4$ dimensions, as shown by Andersson and Edgar (1995). 
Once again, it is remarkable that this very complicated non-linearity for Lanczos potentials of Weyl tensors disappears only in four dimensions.

These calculations and results in this Section are presented in more detail in Höglund (1995); they were derived with the computing techniques described there, and checked with MathTensor, (Parker and Christensen (1991). 


\section{Some comments on Dolan and Kim (1994b).}

The purpose of the second paper by Dolan and Kim was to propose some possible Lanczos potentials in Lanczos gauges for Weyl tensors in some particular spacetimes, and to test these potentials in the LanczosIllge vacuum wave equation (1.8). They made use of some results by Novello and Velloso (1987) who have constructed Lanczos potentials for Weyl tensors in a number of different classes of perfect fluid spacetimes. We emphasise that the work by Novello and Velloso involved lengthy calculations whereby they showed directly that their proposed Lanczos potentials gave rise to genuine Weyl conformal curvature tensors (with the required differential as well as algebraic properties.)

As noted in the Introduction the choices (1.4) are optional gauge choices. However, it is emphasised that in Dolan and Kim (1994b) these Lanczos gauges are always implied, and so it should be understood that when Dolan and Kim talk about a 'Lanczos potential' they are by definition requiring that it is a potential of the Weyl curvature tensor and satisfies both Lanczos gauges. In fact, they construct, where necessary, their potentials in the form (1.13) to guarantee that the algebraic Lanczos gauge is satisfied automatically; no such simple adjustment is possible for the differential gauge condition, so in Dolan and Kim (1994b) the condition (1.4b) is always understood to be supplementing the defining equation (1.11). Obviously, a change of algebraic or differential gauge has no effect on the defining equation (1.11). To avoid misunderstandings in this present paper we shall specifically state when the Lanczos differential gauge is also being required.

In Novello and Velloso (1987) a number of different constructions for Lanczos potentials of Weyl tensors are proposed; when these are applied to a variety of spacetimes, in some cases the Lanczos potential turns out to satisfy the Lanczos algebraic and/or differential gauge. As they pointed out the simple modification (1.13) will always guarantee us a Lanczos potential in the Lanczos algebraic gauge. If we also want to know whether the Lanczos differential gauge is satisfied, then we simply test the Lanczos differential gauge condition $(1.4 \mathrm{~b})$ directly. In the case of the different forms of the Lanczos potentials proposed by Novello and Velloso (1987), then any which also satisfy (1.4b) will obviously automatically be Lanczos potentials in the Lanczos differential gauge.

With the above in mind, we now look in detail at some points from Dolan and Kim (1994b):

\subsection{Possible Lanczos Potentials in the Lanczos Gauges.}

In Section 2 of Dolan and Kim (1994b) three classes of 'possible Lanczos potentials [in Lanczos gauges]' are proposed for Weyl tensors; we consider each of these in detail, and then the respective applications.

(i) The first of their classes is for vacuum spacetimes admitting a hypersurface orthogonal timelike Killing vector $\xi$ - the timelike version in case (a) of Section 2 in Dolan and Kim (1994b) - and as shown there, this implies that the associated unit vector is hypersurface orthogonal, shear-free and expansion-less; so this is just a subclass of the perfect fluid spacetimes containing a hypersurface orthogonal, shear-free timelike unit vector considered in Lemma 2 by Novello and Velloso (1987). Dolan and Kim propose exactly the same form of potential as in Novello and Velloso (1987), and since it was verified there, for the whole class, that 
such a potential is a genuine Lanczos potential in both Lanczos gauges, then clearly the subclass proposed in Dolan and Kim (1994b) also satisfies these conditions. So therefore there is no need for any further verification. We emphasise this point because in Dolan and Kim (1994b) it is stated ${ }^{\dagger}$ 'the [possible Lanczos potential] satisfies (1.2) and (1.4a), and if in each application we verify (1.11a) and (1.4b) then the [possible Lanczos potential] is a Lanczos potential tensor [in Lanczos gauges] for the space-time'; clearly this additional individual verification is unnecessary.

(ii) The third of their classes is for vacuum spacetimes admitting a unit hypersurface orthogonal timelike geodesic field with tangent vector $v$ - case (b) in Section 2 in Dolan and Kim (1994b). This is precisely the vacuum subclass of a class considered by Novello and Velloso in Lemma 3(ii), and Dolan and Kim propose exactly the same potential. In Novello and Velloso (1987) it was shown directly that this proposal is a genuine Lanczos potential, but it was not shown there that this potential satisfies either of the Lanczos gauges. So therefore there is no need for any further verification of the defining equation (1.11a), but the two Lanczos gauges $(1.4 \mathrm{a}, \mathrm{b})$ need to be checked explicitly. We emphasise this point because in Dolan and Kim (1994b) it is again stated 'the [possible Lanczos potential] will satisfy (1.2) and (1.4a) and when we verify (1.11a) and (1.4b) then it will be a Lanczos potential tensor [in Lanczos gauges] for the space-time'; but as we have just noted (1.4a) has not been checked explicitly, whereas (1.11a) has been confirmed explicitly in Novello and Velloso (1987). However, in Appendix I we easily show that the Lanczos algebraic gauge (1.4a) is always satisfied; but in general, the Lanczos differential gauge (1.4b) is not. Therefore if we wish to have a Lanczos potential in the Lanczos gauges for this class, we will need to check only (1.4b) individually for each application.

(iii) Their second class of 'possible Lanczos potentials [in Lanczos gauges]' is for vacuum spacetimes admitting a hypersurface orthogonal spacelike Killing vector $\Xi$ - the spacelike version in case (a) of Section 2 in Dolan and Kim (1994b); this is the spacelike counterpart of the first proposal discussed in (i) above, and the proposed new form for the Lanczos potential $L_{a b c}^{(2)}$ is analogous to the form of the Lanczos potential, discussed above in (i). Since this is a new proposal it is then absolutely necessary - either in a generic manner like in Novello and Velloso (1987), or for each individual application for which this form of potential is chosen - to verify explicitly first of all that (1.11a) is satisfied, and secondly that (1.4b) is satisfied. (The form of the proposed potential guarantees that (1.2) and (1.4a) are automatically satisfied.) However, in Dolan and Kim (1994b), these equations are not verified in a generic manner; and although it is stated again 'the [possible Lanczos potential] satisfies (1.2) and (1.4a) and if in each application we verify (1.11a) and (1.4b) then the [possible Lanczos potential] is a Lanczos potential tensor [in Lanczos gauges] for the space-time', the explicit verification for the application to the Bondi metric is not given.

We emphasise that the point being made here is not that we believe the form $L_{a b c}^{(2)}$ for this class is wrong,

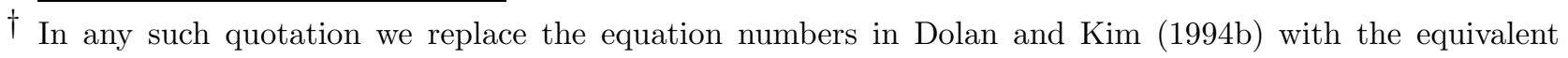
equation numbers in this paper. 
but that there is no explicit proof or statement in Dolan and Kim (1994b) as to why it has been concluded that $L_{a b c}^{(2)}$ is a genuine Lanczos potential of the Weyl tensor of the Bondi spacetime (although an implicit confirmation for the Schwarzschild application is stated).

Since we believe that it has still to be explicitly confirmed that $L_{a b c}^{(2)}$ given for the Bondi metric is indeed a Lanczos potential for the Weyl tensor in the Lanczos gauges, we will discuss this point further and give explicit verification in Section 5 .

(iv) In the remainder of Section 2 of Dolan and Kim (1994b) the three different classes of Lanczos potential in Lanczos gauges are applied to some specific spacetimes. The application (Schwarzschild) chosen to illustrate the class described in (i) above is (as Dolan and Kim note) a spacetime for which an explicit Lanczos potential in Lanczos gauges has already been verified in Novello and Velloso (1987), but we emphasise that this verification was made in Novello and Velloso (1987) for the whole class - they did not have to verify this case individually.

The application (Kasner) chosen to illustrate the class described in (ii) above is (as Dolan and Kim note) a spacetime for which an explicit Lanczos potential in Lanczos gauges has been verified in Novello and Velloso (1987), but we emphasise that its status as a Lanczos potential was verified as part of the whole class they did not have to verify the Lanczos gauge individually.

For the new 'possible Lanczos potential', described in (iii) above, applications are given to both the Schwarzschild and Bondi metrics. There seems some ambiguity in Dolan and Kim (1994b) as to when such a 'possible Lanczos potential' is considered a genuine Lanczos potential. For instance, in the Schwarzschild application in Section 2, a potential of this class $L_{a b c}^{(2)}$, is proposed as a 'possible Lanczos potential', and at this point no justification is given that it is indeed a Lanczos potential of the Weyl tensor in this particular spacetime i.e. whether it satisfies (1.11) - although a comment to this effect is made later, in Section 3. On the other hand, in their next application, also in Section 2, on the Bondi spacetime, it is immediately stated, without any discussion, that $L_{a b c}^{(2)}$ (defined as before) is a Lanczos potential; we feel that this conclusion is premature, since the promised verification in the application to the Bondi metric has not been carried out explicitly, neither for the defining equation (1.11a) nor for the Lanczos differential gauge condition (1.4b).

\subsection{The homogeneous wave equation for the Lanczos potential in Lanczos gauges, in vacuum spacetimes.}

As noted in the Introduction the particularly simple form (1.8) of the wave equation for $L_{a b c}$ occurs in vacuum spacetimes, in the Lanczos gauges. Dolan and Kim set out to provide some 'solutions of this wave equation'. Their procedure is simply to choose some special vacuum spacetime, write down - using examples from each of the three algorithms discussed above - a potential in the Lanczos gauge for that spacetime, and then test explicitly the Lanczos-Illge wave equation (1.8) for that particular Lanczos potential in that particular spacetime. However, for known Lanczos potentials in Lanczos gauges, in vacuum, there is clearly no need to check the wave equation explicitly in each individual case - it follows automatically; calculations 
for individual spacetimes are just special cases of the general case.

On the other hand, for new 'possible Lanczos potentials', the simple wave equation is not guaranteed. However, in Appendix I, when we investigate - as a whole - the new class of possible Lanczos potentials referred to in (iii) above, we discover that all members of this class do in fact satisfy the simple wave equation (1.8). So again there is no need to consider individual cases in this class separately as is done in Dolan and Kim (1994b). In addition, this result becomes very significant when considered alongside our result in Section 4.

\subsection{The Weyl-Lanczos equations.}

Our discussion on this topic relates to both Dolan and Kim (1994b) and to other papers; but we especially wish to draw attention to a structural mistake in the version of these equations in Dolan and Kim (1994b).

(i) The first translations of the Lanczos tensor into spinor notation seems to be due to Maher and Zund (1968), and the first attempt to write down a direct version of (1.11) using NP spin-coefficients, — the five complex Weyl-Lanczos equations - also seems first to have been made in Maher and Zund (1968); however, the notation is unwieldy, and there are a number of errors in that paper. A reliable spinor presentation is given by Taub (1975), and an improved presentation of the Weyl-Lanczos equations, in NP formalism, appeared in Zund (1975) (again with errors, but fewer), together with the NP formalism version (three complex equations) of the Lanczos differential gauge (1.4b). A more reliable version of the Weyl-Lanczos equations is given in Ares de Parga et al. (1989) where there seems to be just one rather obvious misprint - in the expression for $\Psi_{2}$ the coefficient of $\Omega_{3}$ should be $\sigma$ rather than $\alpha$; unfortunately, when some of the same group of authors attempt to quote these equations in a later paper by López-Bonilla et al. (1993) there are a number of misprints.

It is emphasised that in both Zund (1975) and in Ares de Parga et al. (1989) the equations were given in arbitrary differential gauge. Although Zund (1975) uses spinor dyads and Ares de Parga et al.(1989) uses tensor tetrads they essentially agree on their definitions and notation for the eight complex independent components of the Lanczos potential, $\Omega_{0}, \ldots, \Omega_{7}$ - up to a factor of 2 on all components. $\dagger$

(ii) Dolan and Kim (1994b) propose a set of equations - (3.5)-(3.12) — which they also call the 'WeylLanczos equations'. These eight complex equations are a direct NP spin coefficient version of

$$
\Psi_{A B C D}=2 \nabla_{A}{ }^{\dot{A}} L_{B C D}
$$

(which is (1.11b) combined with the Lanczos differential gauge (1.6)) and so consists of the five original Weyl-Lanczos equations - as given in Zund (1975), Ares de Parga et al. (1989) — combined with the

$\dagger$ One has to take care since these two papers use different definitions for the Weyl tensor. Also Ares de Parga et al.(1989) use the conventions of Kramer et al.(1980) for the ordering, numbering and labelling of their tetrad vectors and NP spin coefficients - but with one confusing variation; instead of labelling the tetrad vectors $\{m, \bar{m}, l, k\}$ as in Kramer et al.(1980), they label them as $\{m, \bar{m}, l, n\}$. 
three equations which are the spin coefficient version of the Lanczos differential gauge (1.6). Of course, it is straightforward to separate this set into its two parts. However, there is no mention of the Lanczos differential gauge condition in the section in Dolan and Kim (1994b) on the spin coefficient version; to avoid any misunderstanding this should be noted, so that the more complicated structure of this set of equations be understood. Since this set of NP equations in Dolan and Kim (1994b) include both the defining equations for the Lanczos potential and the Lanczos differential gauge, they alone need to be checked explicitly for new proposed Lanczos potentials in the Lanczos differential gauge.

(iii) When we compare the definitions (in both vector and spinor notation) for the eight complex independent components of the Lanczos potential, $L_{0}, \ldots, L_{7}$ in Dolan and Kim (1994b) with the set of Weyl-Lanczos equations given there - as differential equations for $L_{0}, \ldots, L_{7}$ - we see that there is a systematic inconsistency in sign. ${ }^{\dagger}$ In fact, the definitions of the components of the Lanczos potential, $L_{0}, \ldots, L_{7}$ in Dolan and $\operatorname{Kim}(1994 \mathrm{~b})$ are the negative of the definitions of $\Omega_{0}, \ldots, \Omega_{7}$ in Ares de Parga et al. (1989) and Zund (1975), although the form of the equations in Dolan and Kim (1994b) essentially agrees with the (Lanczos differential gauge version) of the equations in Ares de Parga et al.(1989) and Zund (1975). So we propose that,

1. The definitions (in both tensor and spinor notation) for the eight complex independent components of the Lanczos potential, $L_{0}, \ldots, L_{7}$ in Dolan and Kim (1994b) all be adjusted by a factor of $(-1)$, but their Weyl-Lanczos equations remain unchanged.

2. The values of all explicit Lanczos potential tetrad/dyad components be adjusted by a factor of (-1); in particular this applies to the Section 3 examples for the Schwarzschild and Bondi spacetimes.

These changes will not only correct Dolan and Kim (1994b), but will be consistent with the definitions and equations in Ares de Parga et al. (1989) (and with Zund (1975), subject to the factor of 2, and the misprints there). We shall assume this correction for the rest of this paper, and correct their applications in (iv) below, and in Section 5 .

(iv) The application for the Schwarzschild example — in Section 3 of Dolan and Kim (1994b) — makes explicit use of their 'Weyl-Lanczos equations', and rather surprisingly — in view of the fact that we now know that these equations are inconsistent with the definitions of the Lanczos potential components as proposed in Dolan and Kim (1994b) — it is claimed that their Weyl-Lanczos equations are satisfied. It is certainly true that when the given Lanczos potential components are substituted into their given Weyl-Lanczos equations — for both of the proposed Lanczos potentials $L_{a b c}^{(1)}, L_{a b c}^{(2)}$ — we do find the only non-zero component of the Weyl tensor is $\Psi_{2}=m / r^{3}$, as stated in Dolan and Kim (1994b). However, in the notation and conventions of Penrose and Rindler (1984), the value should be $\Psi_{2}=-m / r^{3}$. (This value can easily be confirmed by

$\dagger$ We believe that this is due to a mistake in sign when lowering an index (or a departure from the conventions of Penrose and Rindler(1984)) going from equation (3.2) to (3.3) in Dolan and Kim (1994b). 
substituting the spin coefficients and differential operators as given by Dolan and Kim (1994b) into the relevant NP equation in Penrose and Rindler (1984)). Hence we find that the calculations in this application do not verify the Weyl-Lanczos equations for the definitions of the Lanczos potential components as proposed in Dolan and Kim (1994b); this is of course as we would now expect, and it is clear that the adjustment by a factor of $(-1)$, proposed above, on all the Lanczos potential components, will ensure agreement.

This corrects the confirmation by direct calculations that $L_{a b c}^{(2)}$ is a genuine Lanczos tensor in the Lanczos gauge for the Schwarzschild metric.

\subsection{The Lanczos-Illge wave equation as a condition for a Lanczos potential.}

Finally we would point out that one reason for stressing these points in detail is the fear - since Dolan and Kim do not in most cases confirm explicitly the defining equation (1.11a) and the Lanczos gauge condition (1.4b), but rather concentrate on the homogeneous Lanczos wave equation (1.8) - that some readers may mistakenly conclude that the homogeneous wave equation is a sufficient (as well as a necessary) condition for a Lanczos potential of the background Weyl tensor in the Lanczos gauges in vacuum spacetimes. There is of course no reason, at this stage, to suspect this; on the other hand we shall show in the next section that this is often the case. 


\section{Lanczos-Illge wave equation as a sufficient condition for Lanczos potential.}

If the simple homogeneous wave equation (1.8) for the Lanczos potential $L_{a b c}$ of the Weyl curvature tensor is going to play a significant role in general relativity, or even in differential geometry, then it is essential to be able to distinguish between generic potentials for any Weyl candidate satisfying Bianchi-like equations, and the Lanczos potentials for Weyl curvature tensors/spinors.

As pointed out in Section 1, Illge (1988) has shown that any Weyl candidate (fully symmetric) spinor $W_{A B C D}$, can always be given locally in terms of a four-index spinor potential $L_{A B C \dot{A}}$ (which is completely symmetric in its three undotted indices) as

$$
W_{A B C D}=2 \nabla_{D}{ }^{\dot{A}} L_{A B C \dot{A}}
$$

in the Lanczos differential gauge

$$
\nabla^{A \dot{A}} L_{A B C \dot{A}}=0
$$

Further, if the Weyl candidate satisfies

$$
\nabla^{D \dot{A}} W_{A B C D}=0
$$

it necessarily follows that its potential satisfies

$$
\square L_{A B C \dot{A}}=0
$$

in vacuum.

The Lanczos potential of the Weyl conformal spinor $\Psi_{A B C D}$, by virtue of the Bianchi equations, also necessarily satisfies (4.4) in vacuum, in the Lanczos gauge. At first sight it would appear unlikely that (4.4) is also a sufficient condition for a potential to be the Lanczos potential of the Weyl conformal spinor of the background vacuum spacetime, but we shall show that this is essentially true.

Although we have no reason to expect an arbitrary Weyl candidate $W_{A B C D}$ satisfying (4.3) in a vacuum spacetime to be the Weyl spinor of the background vacuum spacetime, nor indeed of any spacetime, of course one of these Weyl candidates is the Weyl spinor of the background spacetime. However, Bell and Szekeres (1972) have shown - in a given background vacuum spacetime of sufficient generality - that (4.3) has a unique solution up to a constant factor. Since the Weyl spinor $\Psi_{A B C D}$ of the background spacetime also satisfies (4.3) then - in general circumstances -

$$
\Psi_{A B C D}=k W_{A B C D} \quad \text { where } k \text { is constant }
$$

Therefore, if in a vacuum spacetime, we construct a Weyl candidate by (4.1) from a potential satisfying (4.2) and (4.4) then, in general, that Weyl candidate is actually the Weyl conformal spinor of the background vacuum spacetime (up to a constant factor).

Summing up: In a vacuum spacetime of sufficient generality, equations (4.2) and (4.4) are sufficient conditions that the potential $k L_{A B C \dot{A}}$ be a Lanczos potential for the Weyl curvature spinor given by $\Psi_{A B C D}=$ $k \nabla_{D}{ }^{\dot{B}} L_{A B C \dot{B}}$ of the background vacuum spacetime, where $k$ is a constant factor to be determined. 
There are two distinct special situations when (4.3) fails to have a unique solution (up to a constant factor) and hence our result does not hold:

(a) the background vacuum spacetime is algebraically special;

(b) the background vacuum spacetime is algebraically general but satisfies a very restrictive condition, given explicitly in Bell and Szekeres (1972). For this class, the solutions to (4.3) are linear combinations of, at most, two independent solutions. Therefore it is possible, for this special class, that a solution of (4.4) would give a Weyl candidate which is completely independent of the Weyl spinor.

It should be noted that it has been shown explicitly in Bell and Szekeres (1972) that there is a nontrivial additional term introduced into (4.5) for the exceptional case (a), and it has also been shown that case (b) is not empty by the construction of a counterexample to (4.5). This means that it is not just that we have been unable to prove our result for the Lanczos potential in these two exceptional cases, but that in these cases our result cannot be proven.

Finally, we emphasise that not only are the results in this section valid only in four dimensions but - since the uniqueness result of Bell and Szekeres has been derived using spinors - the results are only valid in spacetimes, with Lorentz signature; it remains to be seen whether these results can be generalised to other signatures and dimension. 


\section{Verification that the new proposal in Dolan and Kim (1994b) gives a Lanczos potential for certain vacuum spacetimes.}

We consider explicitly the proposal in Dolan and Kim (1994b) for a 'possible Lanczos potential' $L_{a b c}^{(2)}$ built around a spacelike Killing vector. Although we believe that it is straightforward to show, in a generic manner, that the defining equation (1.11a) holds in vacuum — by a proof formally analogous to that given by Novello and Velloso (1987) for the timelike case, but of course without the physical interpretations — we shall instead avoid these long calculations and use the result from the last section.

We have shown in Appendix I, that the Lanczos differential condition (1.4b) and the homogeneous wave equation (1.8) do hold for a generic potential $L_{a b c}^{(2)}$ of this class. Therefore the result in the last section immediately verifies that $L_{a b c}^{(2)}$ is indeed a Lanczos potential in the Lanczos gauge - providing our background spacetime is vacuum, and does not fall into one of the two exceptional cases.

We cannot use this result for the Schwarzschild application (since the spacetime is algebraically special), but we have already verified $L_{a b c}^{(2)}$ directly for this case in part (iv) in Section 3.4.

We turn next to the other application of this class given in Dolan and Kim (1994b). Although the application, in Dolan and Kim (1994b), to the Bondi space-time, makes no mention of any vacuum conditions being imposed on the metric functions $U, V, g, b$, we assume that it was intended for the vacuum conditions to be understood. However, it will be interesting and instructive to speculate whether this Lanczos potential is also valid for any non-vacuum Bondi spacetime. ${ }^{\dagger}$ We consider the metric as quoted in Dolan and Kim (1994b), together with the vacuum equations in Di Prisco et al. (1987).

First we consider the vacuum case. Since we know that the vacuum Bondi spacetime has a very general form which does not fall into any of the two exceptional cases in the last section, we can conclude from our result in the last section that the potential proposed for the vacuum Bondi spacetime in Dolan and Kim (1994b) is a genuine Lanczos potential in the Lanczos gauge for the Weyl curvature tensor, up to a constant factor. Turning now to the non-vacuum case we need to test directly whether each component of the Weyl curvature tensor $\Psi_{i}$ calculated in the usual way from the NP equations, agrees with the corresponding component for the Weyl curvature tensor $\Psi_{i}^{L}$ calculated from the proposed Lanczos potential. This is a long but straightforward calculation by hand; but we did it very easily using Maple.

We begin with the simplest component; using the explicit expressions for the spin coefficients in Dolan and

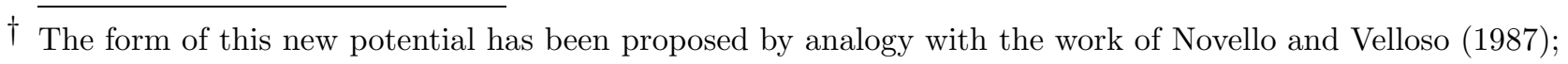
although they considered the more general perfect fluid spacetimes, this was not a necessary constraint on their work and they stated that their results could be generalised further to other non-vacuum spaces. Since Dolan and Kim construct their new potential around a spacelike vector - analogous to the timelike vector used by Novello and Velloso - the results of Dolan and Kim do not generalise obviously in the same manner to perfect fluids; however, a generalisation to perfect fluids or other non-vacuum cases is not explicitly ruled out. 
Kim (1994b) and the usual NP equations, (Newman and Penrose (1962)), we obtain

$$
\begin{aligned}
\Psi_{0} & =D \sigma-\sigma(\rho+\bar{\rho}) \\
& =-e^{-4 b}\left(r g_{11}-2 r b_{1} g_{1}+2 g_{1}\right) / r
\end{aligned}
$$

where the symbols have the meanings given in Di Prisco et al. (1987). On the other hand, using the WeylLanczos equations and the explicit expressions for the Lanczos potential (corrected by a factor of $(-1)$ ) in Dolan and Kim (1994b), we obtain

$$
\begin{aligned}
\Psi_{0}^{L} & =2(D-\bar{\rho}) L_{4}-6 \sigma L_{1} \\
& =-e^{-4 b}\left(r g_{11}-2 r b_{1} g_{1}+2 g_{1}+2 b_{1}-r g_{1}^{2}\right) / r
\end{aligned}
$$

Clearly the $\Psi_{0}$ 's do not agree in general, but it is straightforward to confirm that they agree when we make use of the field equation

$$
\Phi_{00}=e^{-4 b}\left(2 b_{1}-r g_{1}^{2}\right) / r
$$

in vacuum. (This is in fact the first of the main vacuum field equations from Di Prisco et al. (1987)).

Carrying through the same calculations for all five Weyl tensor components we obtain

$$
\begin{aligned}
& \Psi_{0}=\Psi_{0}^{L}+\Phi_{00} \\
& \Psi_{1}=\Psi_{1}^{L}+\Phi_{01} \\
& \Psi_{2}=\Psi_{2}^{L}+\frac{2}{3} \Phi_{11}+\frac{1}{3} \Phi_{02} \\
& \Psi_{3}=\Psi_{3}^{L}+\Phi_{12} \\
& \Psi_{4}=\Psi_{4}^{L}+\Phi_{22}
\end{aligned}
$$

Therefore we find that in order for all the Weyl-Lanczos equations to be satisfied we need to use almost all the vacuum field equations; in particular we find that the most general form of the energy-momentum tensor is

$$
T_{a b}=\alpha\left(l_{(a} n_{b)}-x_{a} x_{b}\right)-\beta \Xi_{a} \Xi_{b}+\gamma g_{a b}
$$

where $\Xi_{a}\left(=r e^{-g} \sin \theta\left(m_{a}-\bar{m}_{a}\right) / \sqrt{2} i\right)$ is the spacelike Killing vector around which the Lanczos potential is constructed and $x_{a}=\left(m_{a}+\bar{m}_{a}\right) / 2 ;\left\{l_{a}, n_{a}, m_{a}, \bar{m}_{a}\right\}$ are the usual null tetrad vectors given in Di Priso et al. (1987), and $\alpha, \beta, \gamma$ are arbitrary scalars. Therefore we cannot use this form for the Lanczos potential in many physically interesting non-vacuum Bondi spacetimes (e.g. perfect fluids, radiation, electomagnetism). However, from the form in (5.5) we see we can generalise to 'vacuum' spacetimes where we permit a nonvanishing cosmological constant, or equivalently to very special perfect fluids.

Finally we point out that the argument given above for the vacuum case left the freedom of a constant factor; once we have confirmed one of the components we can conclude that the value of the constant is unity. (Of course, if we prefer not to use the general result in the previous section to establish the vacuum case, we now have a direct proof for the special case of the Bondi metric.) 


\section{APPENDIX I}

We consider first the class of spacetimes and Lanczos potential considered in Lemma 3(ii) by Novello and Velloso (1987), in case (b) by Dolan and Kim (1994b), and summarised in (ii) in Section 3 of this paper. The potential has the form

$$
L_{a b c}=\frac{1}{3}\left(\sigma_{c a} v_{b}-\sigma_{a b} v_{c}\right)
$$

and using the properties given in Novello and Velloso (1987), we can show by direct substitution

$$
\begin{aligned}
L_{a b}{ }^{a}= & \frac{1}{3}\left(\sigma^{a}{ }_{a} v_{b}-\sigma_{a b} v^{a}\right) \\
= & 0 \\
L_{a b}{ }^{c} ; c= & \frac{1}{3}\left(\sigma_{c a}{ }^{; c} v_{b}-\sigma_{c b}{ }^{; c} v_{a}+\sigma_{c a} v_{b}{ }^{c c}-\sigma_{c b} v_{a}{ }^{; c}\right) \\
= & \frac{1}{3}\left(\sigma_{c a}{ }^{; c} v_{b}-\sigma_{c b}{ }^{; c} v_{a}+\sigma_{c a}\left(\sigma_{b}{ }^{c}+\theta h_{b}{ }^{c} / 3\right)-\sigma_{c b}\left(\sigma_{a}{ }^{c}+\theta h_{a}{ }^{c} / 3\right)\right) \\
= & \frac{1}{3}\left(\sigma_{c a}{ }^{; c} v_{b}-\sigma_{c b}{ }^{; c} v_{a}\right) \\
= & \frac{2}{9}\left(\theta_{, a} v_{b}-\theta_{, b} v_{a}\right)
\end{aligned}
$$

Obviously the Lanczos algebraic gauge is always automatically satisfied, but the Lanczos differential gauge is not always satisfied for all possible spacetimes in this class.

We consider next the new proposal in Dolan and Kim (1994b) — the class of vacuum spacetimes admitting a hypersurface-orthogonal space-like Killing vector $\xi_{a}$. As pointed out in Dolan and Kim (1994b) the associated unit vector $U_{i}=\xi_{i} / \xi$ satisfies

$$
\begin{gathered}
U_{; i}^{i}=0 \\
U_{(i ; j)}\left(\delta_{a}^{i}-U^{i} U_{a}\right)\left(\delta_{b}^{j}-U^{j} U_{b}\right)=0 \\
U_{i ; j}=-A_{i} U_{j} \quad \text { where } \quad A_{i}=U_{i ; j} U^{j}=-(\ln \xi)_{, i}
\end{gathered}
$$

In addition, we note that

$$
\begin{gathered}
U^{i} A_{i}=0 \\
\square U_{i}=-U_{i} A_{j} A^{j} \\
-\square \ln \xi=A_{; i}^{i}=U_{; j i}^{i} U^{j}+U_{; j}^{i} U^{j}{ }_{; i}=0 \\
\square A_{i}=-(\square \ln \xi)_{, i}=0
\end{gathered}
$$

in vacuum.

Dolan and Kim have proposed as a possible Lanczos potential

$$
L_{i j k}^{(2)}=\left(A_{i} U_{j}-A_{j} U_{i}\right) U_{k}+\frac{1}{3}\left(A_{i} g_{j k}-A_{j} g_{i k}\right)
$$


and this clearly satisfies the Lanczos algebraic gauge. By direct substitutions, using the properties listed above, we easily obtain

$$
L_{i j k}^{(2) ; k}=0
$$

and

$$
\square L_{i j k}^{(2)}=0
$$

so the Lanczos differential gauge and the simple wave equation are satisfied for the class of potentials given by $L_{i j k}^{(2)}$. 


\section{Acknowledgements.}

This work was supported, in part, by the Swedish Natural Science Research Council.

\section{REFERENCES.}

Andersson, F. and Edgar, S. Brian (1995). The wave equations for the Weyl tensor/spinor and dimensionally dependent tensor identities. Preprint, Department of Mathematics, University of Linköping, Sweden.

Ares de Parga G., et al (1989) J.Math. Phys., 30, 1294.

Atkins, W.K. and Davis, W.R. (1980). Il Nuovo Cimento, B59, 116.

Bampi, F., and Caviglia, G. (1983). Gen. Rel. Grav.,15, 375.

Bell, P and Szekeres P. (1972). Int. J. Theor. Phys., 6, 111.

Di Prisco, A., Herrera, L., Jimenez, J., Galina, V. and Ibanez, J. (1987). J.Math.Phys., 28, 2692.

Dianyan, Xu (1986) Phys. Rev. D, 35, 769

Dolan, P. and Kim, C.W. (1994a). Proc. R. Soc. Lond. A, 447, 557.

Dolan, P. and Kim, C.W. (1994b). Proc. R. Soc. Lond. A, 447, 577.

Edgar, S. Brian (1994a). Mod. Phys. Lett. A, 9, 479.

Edgar, S. Brian (1994b). G.R.G., 26, 329.

Edgar, S. Brian and Höglund A. (1995) Dimensionally dependent identities for Curvature and Lanczos tensors. Preprint, Department of Mathematics, University of Linköping, Sweden.

Fulling, S A et al. (1992). Class. Quant. Grav., 9, 1151

Hammon K.S. and Norris L.K. (1993) Gen. Rel. Grav., 25, 55.

Harvey, A. (1995). J. Math. Phys., 36, 356

Höglund, A. (1995) "The Lanczos potential and its wave equation.", Preprint, LiTH-MAT-Ex-95-08. Department of Mathematics, University of Linköping, Sweden.

Illge, R. (1988). Gen. Rel. Grav., 20, 551.

Illge, R. (1995). Private Communication.

Jack, I. and Parker, L. (1987). J. Math. Phys., 28, 1137

Kramer, D., Stephani, H., Herlt, E., and MacCallum, M. A. H. (1980). Exact solutions of Einstein's field equations, Cambridge University Press.

Lanczos, C. (1962). Rev. Mod. Phys., 34, 379.

López-Bonilla, J.L. et al (1993) Class. Quant. Grav., 10, 2153

Lovelock, D. (1970). Proc. Camb. Phil. Soc., 68, 345

Maher, W.F. and Zund, J.D. (1968). Il Nuovo Cimento, Serie X, 57 A, 638.

Newman, E.T., and Penrose, R. (1962). J.Math.Phys., 3, 566.

Novello, M and Velloso, V. (1987). Gen. Rel. Grav., 19, 1251.

Parker L. and Christensen S.M. (1991). MathTensor, MathSolutions Inc., Chapel Hill. 
Penrose, R. and Rindler, W. (1984). Spinors and Spacetime Vols.1 and 2 (Cambridge University Press) . Roberts, M.D. (1989). Mod. Phys. Lett. A, 4, 2739.

Taub, A.H. (1975). Comp. \& Math. Appl., 1, 377

Torres del Castillo, G.F. (1995) J.Math.Phys., 36, 195.

Zund, J.D. (1975). Ann. Math.Pura Appl., 104, 239. 